\title{
Recontextualising the style of naming in nomenclature
}

\author{
Paul Rummy (1D ${ }^{1,2,3 凶}$ \& Jessica Thevamalar Rummy (D) ${ }^{4}$
}

In this paper, we brought compelling clarities as to why the current practices of binomial nomenclature should be revised and adjusted by the scientific governing bodies. We highlighted the current emphasis on Latinisation and Greek forms for scientific names has given fewer possibilities for the inclusion of cultural and native scientific names. With regard to zoological matters, we further pointed out that the International Commission on Zoological Nomenclature (ICZN) has an obligation to rationalise the applicability and suitability of the existing Articles and Recommendations in the Code. The Code has been designed to assist scientists in naming an organism with its guidelines. However, the practicality of the Articles and Recommendations need to be further explained in order to reduce several misperceptions within the scientific community; which include the correct usage of Latinised and Greek language forms that can be quite confusing to those who are not well-versed in the structures. The discussion also underlined the novelty of having more localised and hybridised scientific names, and the necessity to avoid norms of abusive, offensive and colonising names since the Code did not emphasise enough on the level of integrity needed with the naming procedures. We further illustrated the magnitude of having a gender-neutral naming system in the world of nomenclature, as the current practices of Latinised and Greek forms are heavily navigated towards masculine naming styles. We also suggest that the noncompulsory Recommendation section of the Code to be made relevant, and perhaps mandatory in some cases, with an infinite approach to accentuate beyond inclusivity and diversity.

\footnotetext{
${ }^{1}$ Key Laboratory of Vertebrate Evolution and Human Origins, Institute of Vertebrate Paleontology and Paleoanthropology, Chinese Academy of Sciences, Beijing, People's Republic of China. ${ }^{2}$ Chinese Academy of Sciences Center for Excellence in Life and Paleoenvironment, Beijing, People's Republic of China. ${ }^{3}$ College of Earth and Planetary Sciences, University of Chinese Academy of Sciences, Beijing, People's Republic of China. ${ }^{4}$ Elintist Arc Research Consultancy, Kuantan, Pahang, Malaysia. ${ }^{凶}$ email: paulrummy@ivpp.ac.cn
} 


\section{Introduction}

ilman and Wright (2020) have conclusively emphasised that it is time for the scientific community to begin a novel stance in how we observe the prominence of preserving cultural names and embracing species-epithets. The authors raised the possibilities to re-establish Indigenous names in nomenclature and include the participation of Indigenous scientists in the process of productive discussions and constructing collective decision makings when it comes to species naming. This is due to the fact that the current practices of nomenclature need to be more relevant and inclusive in the $2020 \mathrm{~s}$ era, and not hold on to years of unchanged procedures by following the Latin and Greek formats. Furthermore, the contemporary principles and practices of nomenclature have always been a westernised pride that has overruled modern sciences with several colonising names, which only proves that the system is very much flawed and requires improvements. Thus, debates have been intensifying in the scientific community with these aforementioned issues, and placing the credibility of the supervision bodies for nomenclature in dire need of an overhaul. We also deem that the changes are inevitable with regard to global context, where equality on every level are being emphasised more than ever. Hence, this paper aims to discuss why the existing practices of nomenclature need to be more inclusive with the integration of cultural, native, localised and hybridised names; why integrity is needed for sensitivity, and also why the present gender bias naming should be revamped in order to create pathways for a more gender-neutral scientific terms. We posit that this discussion would be a motivating factor to gradually shift the traditional convictions among scientists as well. Furthermore, what makes a good solid science, if there is no chamber for enhancements and criticisms?

More so, the art of scientific naming has remained rigid for an extended duration of time. Naming is a vital tool for humans to communicate and convey information in order to help the sciences to grow, the community to flourish and live harmoniously, and reputable relationships to be established as well. Through the lenses of Tippo and Stearn (1977), names can be described as a guiding factor, historically linked and become ingrained in our daily lives. Also, the authors stated that names can initiate relevant groupings and positions for the intended subjects. Moreover, such a fundamental scientific pillar of nomenclature was even well understood by Lewis Carroll (1897), as he has portrayed his ideologies of names in the children's fictional story book, Through the Looking Glass, which has been perceived to be a significant move that has entranced the sphere of classical literature and imagination. This illustrates that the practice of naming is also significant in the world of literature as well.

Nevertheless, as previously mentioned, despite the uniqueness that names possess, numerous traditional scientific scholars have revolved themselves around the custom of using Latinised and Greek forms, a practice that can be seen as a notion of linguistic imperialism with the classical European languages. However, in certain cases, several scientists have been breaking the narratives by utilising vernacular forms instead; to which those distinctive arrangements can be deemed to contain more substantial intrinsic values, due to the fact that they personify a sense of ethnicity, identity and belonging to their sociocultural symbols, local dynamics, indigenous familiarity, mystical myths and legends. These nonconformist scientists have been diligently constructing routes by becoming changing agents for unorthodox naming styles to be integrated and embraced, such as having localised, native or hybridised names that incorporate both the Latin and local languages, an act that is seen to heavily navigate towards cultural relevance and native language maintenance.
Hence, this paper is asserting a grassroot outlook for more alternatives to be made in terms of species nomenclature.

Also, we cannot deny that the independence and autonomy to name species can actually permit scientists to be able to convey their linguistic expressions, personalities or honour a person. In this commentary, we are focusing on binomial nomenclature, or the Linnaean binomial system. Within the same context, it is important to highlight that gender agreement issues in scientific naming have also prevailed for a long time, a matter that can lead to unresolved and ceaseless biases and discriminations. In fact, Guenée (1857) pondered upon the scenario of having to alter names in favour of gender as being equivalent to nonchalantly immature and ignorant to the higher grounds of science, such as having to first understand what constitutes taxonomy and what defines a species level, while some are questioning if species levels really exist.

Notably, it is not our primary intention to evaluate and criticise the rulings in the International Code of Zoological Nomenclature (Ride et al., 1999) [herein, to be referred to as the Code], which uses and facilitates Linnaean binomial system. Rather, we believe that it should be given a rigorous review if the usage of Latinised forms and gender-based styles are still relevant, practical and functional in the taxonomic field. We are also urging scientists to be able to bridge the linkage between communication and the sciences, and how these two variables could impact society's transformation and mindsets. We greatly posit that the freedom to sensible naming will allow for more enhanced communication and connections, followed by reshaping the diplomacy within the multilingual and multicultural scientific societies.

\section{A one size fits all policy for naming: Dilemma or superiority?}

We acknowledge and vastly respect the fact that regulating bodies exist for a precise motive, and such institutions have supreme power in sustaining the present standard practices as scientific names have been designed to be distinguishable in their own distinctive manner for decades. However, it has been brought to our attention that not all protocols would appeal to every context. In fact, the current scientific naming system is creating divisions and distances, and has the potential to continue reinforcing inequality between scientific organisations across the world. In other words, the "one size fits all" policy needs to be stringently and uncompromisingly re-evaluated to allow for more possibilities of local inclusions and Indigenous rights. Also, it has been argued that binomial nomenclature has brought forward astounding confusions in many, not only in language aspects with the complex Latin and Greek grammar, but also in the level of integrity in some of its existence; to which several of them can be likened to as being remarkably offensive, insensitive and nonsensical. An example of an offensive name, which arguably is an inaccurate portrayal of a bird species is the Macronus gularis (Horsfield, 1822), or otherwise known as 'striped tit-babbler', to which the reasoning behind that name cannot be unravelled. The existence of such kinds of names is due to that fact that the Code did not place great emphasis on integrity in naming, but rather place the rules for integrity as a Recommendation (25C) to be followed. It is important to note that the Recommendation section in the Code has not been regarded as obligatory, but rather as mere guidance and suggestions to authors for a preferred way of reciprocal communication. For instance, the Recommendation Article 25C excerpt suggest:

'Authors should exercise reasonable care and consideration in forming new names to ensure that they are chosen with their subsequent users in mind and that, as far as possible, 
they are appropriate, compact, euphonious, memorable, and do not cause offence'...

to which we question why can't the above act be made as mandatory? Isn't our society is still very much governed by solidarity and formality? Having said that, we believe that it is time that certain relevant Recommendations be made compulsory as part of the Code, to which otherwise would remain largely ignored. Ultimately, we ask ourselves where do we draw the line between the act of conforming and non-conforming with the naming regimes? With that, it is noteworthy to declare that there are certain sociolinguistic rules that have to be observed for a polite acceptance that also function in a universally coherent style, which could then be successfully diffused cross-culturally. Meanwhile, it has been said that in the naming of nomenclature, the objectivity of using Latinised or Greek forms was to reduce the confusions with common names and to avoid the existence of synonyms, although at times synonym's mistakes are inexorable as the library of new named species has been widely increasing throughout the years.

At present, we deem that scientific names should exist as one name, and synonyms or homonyms will cause issues and unwanted troubles. In the case of a venomous snake bite, searching for the right name of the snake genus may be crucial in administering anti-venom in order to save lives. However, the snake species name of Spracklandus (Hoser, 2009) for example is rarely used by taxonomists and is more commonly known as Afronaja (Wallach et al., 2009) instead, as mentioned by Jones (2017). Despite that as well, such a problem should not be a major concern in an era where giant corporations, institutions and individuals could spend billions of funding in technology, artificial intelligence and initiating the world's next smart generation network of the $5 \mathrm{G}$ and $6 \mathrm{G}$. Hence, the issues of synonyms can be potentially resolved by using online binomial nomenclature registration platforms such as the Integrated Taxonomic Information System (Guala, 2016) and other registering bodies, as it is just a matter of database management. In fact, Vlachos (2019) came up with an effective networking tool to help all levels of users to comprehend the Code in a much easier platform. In a logical perspective as well, most of the scientific research is being conducted and reported in the English language, which has been regarded as the lingua franca of the world. Since it is a burden for some scientists to work on longitudinal, tedious and timesensitive research work, why place them in a complicated and multiplex position by having them face the task of utilising a language that barely exist in the modern times, just for the mere sake of naming a new taxa?

Also, in a more liberating angle, a dead language for Linnaeus was a non-complex notion. It has also been said that Linnaeus used the binomial method to honour his predecessors, however, there has been an occasion where he was presumed to have insulted a fellow friend with a Latin name after a quarrel. In light of such circumstances, it is very apparent that linguistic expressions can sometimes be menacing if used in ambiguous, colonising and abusive manners, or as means to ignite insultations and glorify racism and gender biases. There have been instances where Indigenous species found by western scientists have been named after prominent western Presidents, or white males that are considered to be of a priviledge position, an act that we call as parachute science. On the other hand, with the intentions of wanting to insult, some scientists throughout history have gone on to name newly discovered species after their fellow colleagues, not to honour but to make fun of them. Therefore, again we ask ourselves, where do we draw the lines between patronising the regulations and wanting to have the privileges of free expressions? We would also need to emphasise that the term 'freedom' is not to be largely used against the backdrops of human righteous behaviours, but more towards having the decency to be courteous and considerate of other beings.

Aligned with these thoughts as well, the Code could be regarded as being incapacitated as it does not synchronise when it is required, but remains stagnant and vague for most of the period, without any systemic solutions. Furthermore, if the ICZN is not being explicit about the regulations, there are certainly no boundaries to be followed upon, and neither are there restrictions to the so-called naming creativity. More significantly, Sommerer (2002) highlighted that there is a diverse range of taxonomists with different traditional groundings, therefore a structured repositioning outside of the Latin language has been noticed. Moreover, although there are certain scientists from other cultural heritage who are articulated in the classical language, the plot may not be admissible in the forthcoming decades because even scholars in the European context are having the least command with these dead languages. In fact, the usage of classic Greek is almost nowhere to be found among present day zoologists. Therefore, why limit oneself if there are no such rules? On a separate note, if such rulings have been compulsory, then it should be entirely up to the name givers to uphold the required respect and integrity.

\section{A consideration of non-conformist style}

In this section, we highlight several examples of localised and hybridised scientific names, where these names have been deem as relevant and relatable to local sociocultural dynamics with deep-meanings, but otherwise would not have been if the names were to be in Latinised or Greek forms. The examples highlighted below also indicate how localised and hybridised forms of scientific naming can be effectively used to overcome some of the limitations and constraints associated with the traditional systems of nomenclature.

Among the dinosaur species that were discovered in the Chinese regions, Mei long (Xu and Norell, 2004), Banji long (Xu and Han, 2010), Lingwulong shenqi (Xu et al., 2018), Hexing qingyi (Jin et al., 2012), Caihong juji (Hu et al., 2018) and Qijianglong quokr (Xing et al., 2015) are fully localised binomial nomenclatures that have been given by Chinese palaeontologists, which are all situated within the Code's language regulations if one has understood the forms properly. Taking the Banji long for example, the name has been formed in an adjective-noun format (in accordance to the Code's requirement) and long is the Mandarin term for dinosaurs, which is also another term for 'dragon' in the native language. Besides that, another fully localised name is $Y i$ qi (Xu et al., 2015). The generic name $Y i$ means 'wing' in Mandarin, and the specific name $q i$ means 'strange'. Also, $Y i$ is notable for having the shortest generic name for any records of dinosaurs found, containing only two letters. Its binomial name, Yi qi, is also considerably the shortest under Articles 11.8.1 and 11.9.1 of the Code, at just four letters. Meanwhile, what we defined as a hybridised name is when one part is Latinised or in Greek, while the other part is localised. Beishanlong grandis (Makovicky et al., 2010) is an example of a hybridised name with the generic name being combined with references to 'Bei Shan', or the 'north mountains', followed by long and the Latin term for large, or grandis.

More significantly, we are also observing a trend in full Indigenous names being mobilised, such as the recently discovered orchid species in the Venezuelan territory, which was named as Epidendrum katarun-yariku (Hágsater and Wrazidlo, 2020). Hágsater and Wrazidlo (2020) stated that the 'katarun' refers to 'high', while the word 'yariku' points towards the 'flower', based on the Pemón Arekuna language. The name was chosen based on 
the geographical location of the orchids, which are commonly situated on elevated grounds in the region. On a separate note, there are some names that can be categorised as intriguing, random, humorous and cynical, or unexpected. Arnold Menke (1977) named an Australian wasp as Aha ha and it can be considered as one of the earliest forms of names that was not Latinised since the Code was first introduced. The name Aha ha was considered as a joke for Menke. There were also instances where a species has been named for conservation purposes, such as the GoldenPalace.com monkey in Bolivia, while Carmenelectra shechisme (Evenhuis, 2002) was a pure expression of peculiar creativity.

All the aforementioned names portray and illustrate the meanings that nonconformists wanted to convey, and these certainly gave a whole new dimension of being bona fide, although this is very much an individual preference, respectively. In spite of that, we understand that Latinisation and Greek forms were designed for stability and uniformity. However, when a species is something that is beyond explanation, we will have to learn to accept and exert it accordingly, because taxonomy is all about instability as cladistic relationship is a constant changing hypothesis (Dominguez and Wheeler, 1997). Above all, it is staggering to discover that names that have been deemed as 'derogatory' in a normal scenario are not solid enough to be revoked because there are no ordinances to overturn them in veto. In short, there are no specific guidelines to determine what constitutes a term as being 'derogatory' in the Code, which could easily be brushed away as just mere individual opinions. Imperatively, the present de-colonising movements in scientific research, or 'de-colonising sciences' are being taken seriously by relevant parties, and it would not be detrimental to break one or two menial rules and enrich the nomenclature world with more in-context vernacular, localised, hybridised and Indigenous names.

In addition, the act of parachute sciences should also be avoided. Instead, ethical habits must be cultivated at least to respect and recognise local culture and Indigenous knowledge, a collective mark that can be seen as reducing white supremacy, imperialism and authoritarian behaviours in sciences. Moreover, in a bold and decisive reminder, we should never abuse the opportunities given, to which some have termed this sort of practice as 'taxonomy vandalism' (Naish, 2013) and turn any new rulings into naming absolutism. Why we say so is because any alterations in taxonomy would become extremely difficult, due to the straightforward fact that once the names have been published, it is almost impossible to eliminate them.

\section{Does gender really matter in the 2020s?}

In this section, we will emphasise upon the importance of having gender equality in naming. Several critics have brought up the notion that gender subjects are constantly dominating. The Latin declensions for instance, is a great indicator on how genders are being displayed. Sommerer (2002) also stated that right till the first-quarter of the 19th century, Latin was the go to language for the scientific naming system, which is quite contradictory to the current context as the official functioning language for zoological nomenclature is now English. Sommerer emphasised that the adjectives in English language are not bound by gender for the nouns. The author mentioned that there are risk factors involved if taxonomists are not able to properly and accurately decode the gender agreements. Also, the Code is heavily oriented towards an automatic assumption of masculinity, unless clearly stated by the author that there is an assigned gender for the species. Therefore, do we really need to have genitives in naming? As interesting as it gets, we understood that Linnaeus first proposed this as he was naming plants, which consisted of male and female counterparts. We also understand that if patronym is practiced, there is some logical sense as to wanting to honour a particular person. However, giving a name on the sole purpose of wanting to fit the epithet of Latin grammar may very well be completely irrational, as we could only imagine if someone were to question in the case of a Tyrannosaurus rex (Osborn, 1905), would it be a he, she or neuter? Although in this scenario, rex means 'king' in Latin, which has been placed as a 'he'. Nevertheless, one of the T. rex has a feminine nickname called 'Sue', and the reason behind it was to honour the person who founded it, which was Sue Hendrickson in 1990. However, it appears as the particular T. rex has a masculine and feminine name at the same time, and a genitive declension for it would have been extremely out of place.

Furthermore, the gender concerns not only exist in the nomenclature system, but also within the community itself. Heard (2020) also stated that the Aloe plant genus were found to have a total of 278 eponymous naming, and out of that, $87 \%$ have been oriented towards males, to which Darwin mentioned were predominantly westerners. We wonder if the existence of male gendered names are also due to the fact that the scientific world is run by mostly men. In fact, recent surveys have showcased that the scientific community is being largely dominated by men, as the number of women in scientific publications have recorded a bewildering minimal number, which has solely worsened at the protracted crisis withheld by the pandemic as women became more submerged in domestic matters. Furthermore, this has also extended to the scientific LGBTIQA+ communities, where a proclamation of lack in opportunities and discriminations in research have also been made. These bring us into the question that can the scientific world become completely gender neutral and unbiased? Perhaps this could be seen as something that has been blown out of proportions, however, there is no denying that gender disparities in society and languages can fabricate a hierarchical ranking that echoes status and power. Also, the fact that there are clear differences between male and female representations in the genitive naming could steer towards unequal gender functions in the scientific communities across the world as well. So, we ask how genitive or gender in species and humans would intermingle with social class and sciences? The answer for this question may just be quite intricate to explain, and challenging to be interpreted.

[Intermission: We have specifically mentioned that this commentary is not an assessment or evaluations of the current standard practices in nomenclature. Having said that, it is not our intention to submit any proposals for amendments. Rather, we are choosing to publicise how certain discourse and stylistic representations of the Code can be oscillating and unmodulated to the scientific community. We urge the governing bodies to have solitary measures to enhance the diplomacy of the Code, an act that will ensure diversity and inclusion with scientific names can be acheivable. The Code has gone through an evolution in a duration of 250 years (Dubois, 2011) and currently consist of 90 Articles, with 754 sub-Articles, 129 Recommendations and 129 Examples (Vlachos, 2019). Despite that, the language and structure requirements of the Code when it comes to scientific naming procedures can be difficult for experts that are not familiar with the context, and become more harder with certain conditions applied (Dubois, 2011). The subsequent paragraphs will examine four specific Articles and Recommendations in the Code that need to be given some considerations for alterations, mainly by using linguistics interpretations.]

\section{Deconstructing the discourse of the four rules}

I Recommendation 11A. 'Recommendation 11A: Use of vernacular names: An unmodified vernacular word should not be used 
as a scientific name. Appropriate Latinisation is the preferred means of formation of names from vernacular words.'

As previously mentioned, the Recommendation section of the Code is not mandatory and this is when the confusion arises. The riveting question is, if such is just a recommendation, do scientists have a choice to not compel with this particular ruling? Will it be appropriate to give a vernacular name on one's own terms and conditions then? Also, linguistically, the syntax of the sentence, when combined with the auxiliary verb 'should not' becomes a cryptic interpretation, indicating a gap in the context and pragmatics on whether it can be 'should I?' or 'shouldn't I?'. The uncertainties resume when it was declared that Latinisation of the vernacular forms is the 'preferred' one, a lexical term that is neither definite nor infinite. Therefore, when deconstructing the structure of Recommendation $11 \mathrm{~A}$, it is apparent that there is a contradicting element in the discourse, to which the auxiliary term 'should not' does not contest against the infinite, and with a rather ambiguous term of 'preferred', indicate that there is still an option. Without a doubt, the entire semantics of the Recommendation can be perplexing to those who do not see the relevance in it, and a more straightforward and conclusive approach should be employed in restructuring it.

II Recommendation 25C. 'Recommendation 25C: Responsibility of authors forming new names: Authors should exercise reasonable care and consideration in forming new names to ensure that they are chosen with their subsequent users in mind and that, as far as possible, they are appropriate, compact, euphonious, memorable, and do not cause offence.'

We believe that Recommendation 25C should be ingrained as an Article, and not placed as a Recommendation in the Code. We highly deem that since the responsibility of naming is not being emphasised enough, it has created a space for impertinent and audacious attitudes. As a matter of fact, anyone can publish any names because it is just a recommendation to be courteous and polite, and once those names have been published, it cannot be taken down unless there is a case of synonyms being raised.

This has brought forward our attention to the 'Principle of Priority' in Article 23 of the Code, where it was stated that:

'valid name of a taxon is the oldest available name applied to it'...

This indicates that anything that has been published first can be considered as valid, therefore, changing it is almost non-viable. In light of such syntax issues, if the ICZN chooses to verify the discourse, then we might be able to see a clearer notion in terms of naming ethics and the seniority involved.

III Article and Recommendation 30. 'Article 30.2.3: If no gender was specified, the name takes the gender indicated by its combination with one or more adjectival species-group names of the originally included nominal species [Article 67.2].'

'Article 30.2.4: If no gender was specified or indicated, the name is to be treated as masculine, except that, if the name ends in $-\mathrm{a}$ the gender is feminine, and if it ends in -um, -on, or -u the gender is neuter.'

'Recommendation 30A: Gender and derivation to be made explicit. Authors should expressly state the gender and derivation of a new genus-group name when establishing it.'

'Recommendation 30B: Gender to be made self-evident. So that the gender of new genus-group names is self-evident, authors, when forming new names based on words that are not Latin or Greek and stating their genders, are advised to choose genders for them appropriate to their endings.'
We foresee that these Articles and Recommendations will become increasingly problematic in the coming future, as these are clearly steering towards an unbalanced dynamic in nomenclature, with more species names being propelled to be masculine if not specifically indicated. As a matter of fact, vernacular forms are recommended to follow such agreements if possible as selfproclamation, which should not be the case as certain languages are not bound by gender agreements, as compared to most of the European Languages.

IV Article 31. 'Article 31.1.2: A species-group name, if a noun in the genitive case (see Article 11.9.1.3) formed directly from a modern personal name, is to be formed by adding to the stem of that name -i if the personal name is that of a man, -orum if of men or of man (men) and woman (women) together, -ae if of a woman, and -arum if of women; the stem of such a name is determined by the action of the original author when forming the genitive.'

In contrast to Article 30, we deemed this act of being able to honour a person on the grounds of gender as being appropriate and respectful, and the author will have the choice to identify with their own gender when it comes to naming the species, although we would still highly suggest for a gender-neutral term to be used in future nomenclature.

\section{Conclusion}

Based on the premise that changes are inevitable, we have strived to sketch a picture and depict centuries of narratives that have to be relinquished. We cannot deny that the root of culture is language, and the root of communication is name. Hence, adaptation and assimilation are pivotal in the modern scientific community in order to generate necessary trails for efficacious communication in a timely manner. Also, language has always been a fundamental national identity attribution, an undeniable force of authenticity, to which no culture or civilisation can be sustainable without its existence. Therefore, it should be solicited with due respect and freedom of usage that can contribute to a sense of interconnectedness. Also, it seems like we are collectively facing issues with these current naming styles and therefore, how can we change this current system? Although there is such rich diversity in culture, languages and ethnicities in the world, why are we restricting ourselves to conform to one unanimous method? We uphold the notion that languages and linguistics' applications in nomenclature naming should not be scrambled by stringent rules. In this commentary, we also took into account that having the consensus of everyone can be difficult to achieve as there is still a community of strong, hardcore traditionalists out there, respectfully. But, bearing in mind that all these could be seen as an act of isolation in the making, scientists should begin to be mindful by being accountable, while the governing bodies can most definitely compromise and improvise. With that, we are aware that the Linnaean binomial system is still essential for all scientists out there, but a progressive change can be beneficial. Well, Phylocode? We will have to spare it for another day!

Received: 21 April 2021; Accepted: 4 November 2021; Published online: 18 November 2021

\section{References}

Carroll L (1897[1872]) Through the looking glass and what Alice found there. Henry Altemus Company, Philadelphia

Dominguez E, Wheeler QD (1997) Taxonomic stability is ignorance. Cladistic 13:367-372

Hu D, Clarke JA, Eliason CM, Qiu R, Li Q, Shawkey MD, Zhao C, D’Alba L, Jiang J, Xu X (2018) A bony-crested Jurassic dinosaur with evidence of iridescent 
plumage highlights complexity in early paravian evolution Nat Commun 9(1):217. https://doi.org/10.1038/s41467-017-02515-y

Dubois A (2011) The International Code of Zoological Nomenclature must be drastically improved before it is too late. Bionomina 2:1-104

Evenhuis NL (2002) Review of the Tertiary microbombyliids (Diptera: Mythicomyiidae) in Baltic, Bitterfeld, and Dominican amber. Zootaxa 100:1-15. https://doi.org/10.11646/zootaxa.100.1.1

Gilman LN, Wright SD (2020) Restoring indigenous names in taxonomy. Commun Biol 3:609. https://doi.org/10.1038/s42003-020-01344-y

Guala GF(2016) The importance of species name synonyms in literature searches PLoS ONE 11(9):e0162648. https://doi.org/10.1371/journal.pone.0162648

Guenée A (1857 [1858]) Uranides et Phalénites. In: Boisduval JBAD’E, Guenée A (eds) Histoire naturelle des insectes. Species general des Lépidoptères, Librairie Encyclopédique de Roret, rue Hautefeuille (Paris), vol 9. Généralités. pp. ix-xxxvii

Hágsater E, Wrazidlo M (2020) Epidendrum katarun-yariku (Orchidaceae), a new species of the Schistochilum group from the Tepuis of the Guiana Highlands in South America. Phytotaxa 472(1):33-40. https://doi.org/10.11646/phytotaxa.472.1.4

Heard SB (2020) Charles Darwin's Barnacle and David Bowie's Spider: how scientific names celebrate adventurers, heroes, and even a few scoundrels. Yale University Press, New Haven and London

Horsfield T (1822) Zoological researches in Java and the neighbouring islands. Kingsbury, Parbury and Allen, London

Hoser RT (2009) A reclassification of the rattlesnakes; species formerly exclusively referred to the genera Crotalus and Sistrurus. Australas J Herpetol 6:1-21

Jin L, Chen J, Godefroit P (2012) A new basal ornithomimosaur (dinosauria: theropoda) from the early Cretaceous Yixian formation, northeast China. In Godefroit P (ed.) Bernissart dinosaurs and early Cretaceous terrestrial ecosystems. Indiana University Press. pp. 467-488

Jones B (2017) A few bad scientists are threatening to topple taxonomy. Smithson Mag. https://www.smithsonianmag.com/science-nature/the-big-ugly-problemheart-of-taxonomy-180964629/. Accessed 1 Apr 2021

Makovicky PJ, Li D, Gao K, Lewin M, Erickson GM, Norell MA (2010) A giant ornithomimosaur from the Early Cretaceous of China. Proc R Soc B: Biol Sci 277(1679):191-198. https://doi.org/10.1098/rspb.2009.0236

Menke AS(1977) Aha, a new genus of Australian Sphecidae, and revised key to the world genera of the tribe Miscophini (Hymenoptera, Sphecidae, Larrinae). Pol Pismo Entomol 47:671-681

Naish D (2013) Taxonomic vandalism and the Raymond Hoser problem. https:// blogs.scientificamerican.com/tetrapod-zoology/taxonomic-vandalism-andhoser/. Accessed 25 Mar 2021

Osborn HF (1905) Tyrannosaurus and other Cretaceous carnivorous dinosaurs. Bull Am Mus Nat Hist 21:259-265

Ride WD et al. (1999) International code of zoological nomenclature, 4th edn. International Trust for Zoological Nomenclature, The Natural History Museum

Sommerer M (2002) To agree or not to agree-the question of gender agreement in the International Code of Zoological Nomenclature. Nota Lepidopterol 25:191-204

Tippo O, Stearn W (1977) Humanistic botany. Norton, New York

Vlachos E (2019) Introducing a new tool to navigate, understand and use International Codes of Nomenclature. PeerJ 7:e8127. https://doi.org/10.7717/ peerj.8127

Wallach V, Wüster W, Broadley D (2009) In praise of subgenera: taxonomic status of cobras of the genus Naja Laurenti (Serpentes: Elapidae). Zootaxa 2236:26-36. https://doi.org/10.5281/zenodo.190422

Xing L, Miyashita T, Zhang J, Li D, Ye Y, Sekiya T, Wang F, Currie P (2015) A new sauropod dinosaur from the Late Jurassic of China and the diversity, dis- tribution, and relationships of mamenchisaurids. J Vertebr Paleontol 35:e889701. https://doi.org/10.1080/02724634.2014.889701. S2CID 86062974

Xu X, Upchurch P, Mannion P, Barrett P, Regalado Fernandez O, Mo J, Ma J, Liu H (2018) A new Middle Jurassic diplodocoid suggests an earlier dispersal and diversification of sauropod dinosaurs. Nat Commun 9. https://doi.org/ 10.1038/s41467-018-05128-1

Xu X, Han FL (2010) A new oviraptorid dinosaur (Theropoda: Oviraptorosauria) from the Upper Cretaceous of China. Vertebr PalAsiat 48(1):11-18

Xu X, Norell MA (2004) A new troodontid dinosaur from China with avian-like sleeping posture. Nature 431(7010):838-41. https://doi.org/10.1038/ nature 02898

Xu X, Zheng X, Sullivan C, Wang X, Xing L, Wang Y, Zhang X, O'Connor JK, Zhang F, Pan Y (2015) A bizarre Jurassic maniraptoran theropod with preserved evidence of membranous wings Nature 521(7550):70-73. https:// doi.org/10.1038/nature14423

\section{Acknowledgements}

We truly appreciate X. Xu, Q. Zhao, C.-C. Liao, Z.-C. Qin, C.-Y. Yu, T.-T. Lim and K. Halaclar for the highly intellectual and productive discussions and debates on the issues of binomial nomenclature. Their constructive criticisms and valuable input have greatly set a remarkable mark and guidance on our ends, which have tremendously enabled us to boldly, courageously and decisively steer the direction of this manuscript. P. Rummy is supported by a scholarship from the University of Chinese Academic of Sciences and the National Natural Science Foundation of China (Grant no. 41688103).

\section{Author contributions}

P.R. and J.T.R. equally contributed to the manuscript.

\section{Competing interests}

The authors declare no competing interests.

\section{Additional information}

Correspondence and requests for materials should be addressed to Paul Rummy.

Reprints and permission information is available at http://www.nature.com/reprints

Publisher's note Springer Nature remains neutral with regard to jurisdictional claims in published maps and institutional affiliations.

Open Access This article is licensed under a Creative Commons Attribution 4.0 International License, which permits use, sharing, adaptation, distribution and reproduction in any medium or format, as long as you give appropriate credit to the original author(s) and the source, provide a link to the Creative Commons license, and indicate if changes were made. The images or other third party material in this article are included in the article's Creative Commons license, unless indicated otherwise in a credit line to the material. If material is not included in the article's Creative Commons license and your intended use is not permitted by statutory regulation or exceeds the permitted use, you will need to obtain permission directly from the copyright holder. To view a copy of this license, visit http://creativecommons.org/ licenses/by/4.0/

(C) The Author(s) 2021 